Article

\title{
Ang Landas ng Pagtatanong ni Heidegger ukol sa Teknolohiya: Pagtatalas sa Tanong sa Sein
}

\section{Emmanuel C. de Leon}

\begin{abstract}
This paper attempts to expound the thoughts of Martin Heidegger in the Filipino language. It focuses on an aspect of Heidegger's philosophy which has tremendous relevance to the modern world and the modernizing Philippine context. This paper aims to show the connection between Heidegger's philosophy of technology and his main task of elucidating the Question of Being (Seinsfrage). It argues that Heidegger's rediscovery of the historical truth of Being from the early Greeks brings him to his questions concerning technology. The paper begins with Heidegger's deconstruction of the whole history of Western metaphysics in order to retrieve a kind of thinking that is responsive to the call of Being. It also discusses Heidegger's exposition of the danger of modern technology that challenges nature to produce something for stockfilling and use. His way out of the danger is by creating an ever awake condition on the part of man. Man, as he responds to the call of Being, must retrieve his origin-al role in the destining of Being. He must be watchfull, for sleeping in a bank is a very dangerous vice.
\end{abstract}

Keywords: Heidegger, Being, Filipino language, technology

\section{Panimula}

$0^{2}$ a simula pa lamang, ang pamimilosopiya ni Martin Heidegger ay nakatuon sa paglilinaw ng kahulugan ng tanong tungkol sa Sein (Seinsfrage). Nanggagaling ang proyektong ito sa kanyang pangunahing thesis na ang mismong kasaysayan ng kanluraning metapisika ay isang kasaysayan ng pagkalimot sa tanong sa Sein. Gamit ang kaisipan ng penomenolohiya, binalangkas ni Heidegger ang landas ng katotohanan ng Sein na talagang nangyayari sa iba't ibang panahon ng kasaysayan upang matalastas ang tunay na relasyon ng Dasein sa Sein. Samakatuwid, tinatanong ng papel na ito ang kaugnayan ng pagtatanong ni Heidegger

(C) 2013 Emmanuel C. de Leon

http://www.kritike.org/journal/issue 13/de leon december2013.pdf

ISSN 1908-7330

(cc) BY-NC 
ukol sa teknolohiya at ng kanyang pangunahing adhikain na malinawan ang kahulugan ng tanong tungkol sa Sein.

Layunin ng papel na ito ang maisiwalat ang kaugnayan ng ontolohiya ni Heidegger sa kanyang pilosopiya sa teknolohiya sa pamamagitan ng pagmumuni muni sa daan ng katotohanan na binalangkas ni Heidegger. Ang matapat na pagsama sa masalimuot na pag-iisip ni Heidegger ang diskarte ng papel na ito. Bibigyang diin rin dito ang kapalaran ng pag-iisip ni Heidegger na nauwi sa dead-end at ilang posibleng ruta palabas sa nasabing sitwasyon ng pag-iisip. Harinawa'y dalhin tayo ng mismong kaisipan tungo sa pagkaunawa na bumubukal sa mailap na paglalantad at pagtatago ng katotohanan ng Sein.

\section{Ang Mapanganib na Pagkalimot sa Tanong sa Sein}

Ang salitang "limot" ay tumutukoy sa estado ng hindi pagkaalam sa isang bagay na dati namang nalalaman. Ganito isinalawaran ni Heidegger ang kalagayan ng pag-iisip sa larangan ng metapisika. Sa paningin ni Heidegger, ang kasaysayan ng Kanluraning Metapisika ay kasaysayan ng pagkalimot sa katanungan sa Sein. ${ }^{1}$ Ano ang ibig sabihin ni Heidegger sa akusasyon niyang ito? Kung ang metapisika, sa mayamang kahulugan ng salitang ito, ay ang sangay ng pilosopiya kung saan ang pangunahing proyekto ay ang pagtatanong tungkol sa Sein, bakit nasabi ni Heidegger na ang buong kasaysayan ng kanluraning metapisika ay ang pagkalimot sa mismong suheto nito? At, ano ang sanhi at bunga ng sinasabing pagkalimot na ito sa atin bilang mga tao??

Para kay Heidegger, ang pinaka-tanong ng metapisika, i.e., "Ano ang Sein?," ay itinatanong habang nakakaligtaan ang pag-uusisa sa katotohanan ng mismong Sein. Nangyari ito, ayon kay Heidegger, nang ituon ang pagmemetapisika sa ano ng mga bagay na nakikita sa kanilang pagka-lantad. Samakatuwid, sa paglalagom ni Heidegger, ang bulto ng

${ }^{1}$ Sa "Introduction" ng Being and Time, isinanalaysay ni Heidegger ang mga sintomas ng pagkalimot sa Sein. Sabi niya, "We do not know what 'being' means. But already when we ask, 'What is being?' we stand in an understanding of the 'is' without being able to determine conceptually what the 'is' means." Martin Heidegger, Being and Time, trans. by Joan Stambaugh (New York: State University of New York Press, 1996), 4 (Sa mga susunod, ang mga halaw sa kathang ito ay ilalagay na $B T$ ).

2 May kahirapan ang pagsasalin ng salitang Sein sa wikang Filipino. Maging si Heidegger ay hindi maipaliwanag ang mismong essensya nito. Sa kanyang "Letter on Humanism," tinangka niyang ipaliwanag ang ano ng Sein. Maingat niyang itinanong, "Yet Being - what is Being? It is It itself." Tingnan sa Martin Heidegger, "Letter on Humanism," in Basic Writings, ed. David Farrell Krell (USA: HarperCollins Publishers, 1993), 234. Ipapasya nating gamitin ang orihinal na salitang Sein sa papel na ito upang mapanatili ang yaman at posibilidad ng kahulugan nito.

(c) 2013 Emmanuel C. de Leon

http://www.kritike.org/journal/issue 13/de leon december2013.pdf

ISSN 1908-7330

(cc) BY-NC 
kasaysayan ng Kanluraning metapisika ay ang pagiging istranghero ng tao sa katotohanan ng Sein (Seinsvergenssenheit).

Kung ang pagtatanong na metapisikal ay sinasabi ni Heidegger na pagtatanong ng mga istranghero sa katotohanan ng mismong suheto nito, samakatuwid mayroon ngang orihinal na paraan ng pagtatanong sa metapisika. Ayon kay Heidegger, ang mga sinaunang Griyego ang nakakaalam kung paano magmuni muni sa Sein. Nguni't sa pagdaan ng maraming panahon at pag-usbong ng syentia, ito ay nakalimutan at natuon na lamang sa mga bagay na pwedeng manipulahin at kwentahin. Kaya nga, pinag-ukulan ng pansin ni Heidegger ang "paghakbang-pabalik" sa mga sinaunang Griyego upang bawiin, sa paraang malikhain, ang orihinal na kahulugan ng Sein.

Ang Sein, sa orihinal na pagkaunawa ng mga Griyego, ay nangangahulugang phusis-bilang paraan ng kusang pagbubuyangyang o paglalantad mula sa pagkakatago. Sa paglalantad na ito mula sa pagkakatago, ang Seindes o ang mga bagay bagay ay nananatili sa pagkalantad. Ang pananatiling ito sa pagkalantad (aletheia) ang nagbigay daan para masuri ang mukha o hitsura-ang pagka-ano, na siya rin namang naging sentro ng pagmemetapisika. Ang pagtuon sa pagka-ano ng mga bagay ang naging dahilan upang makalimutan ang pagtatanong sa Sein mismo.

Mahalaga sa pilosopiya ni Heidegger ang kanyang pagmumunimuni sa katotohanan. Ginalugad niya ang tradisyunal na kahulugan ng katotohanan na naging "veritas est adequatio rei et intellectus" 3 o ang tinatawag na correspondence theory of truth. Ang problema sa ganitong konsepto ng katotohanan, ayon kay Heidegger, ay ang kahirapang malaman ang pisikal na relasyon ng simbolo (sinasalita o sinusulat) sa mismong laman ng isip. Nililinawan ni Heidegger na ang salitang katotohanan sa salitang Griyego ay aletheia, na literal na nangangahulugang paglalantad ("unhiddenness"). ${ }^{4}$ Ang paggamit niya ng salita ay nagmumungkahing mayroon lamang katotohanan kung mayroong inilalantad. Samakatuwid, ang katotohanan ay mayroon nang presensya, pero nakatago pa.

Ang Sein bilang aletheia ay isang pangyayari ng pagtatago at paglalantad, isang lantarang-pagtatago. ${ }^{5}$ Nguni't, ang mabagsik na

3 Tingnan sa Martin Heidegger, "On the Essence of Truth," trans. by John Sallis, in Pathmarks, ed. William McNeill (Cambridge: Cambridge University Press, 1998), 138.

${ }^{4} B T, 265 / 222$.

${ }^{5}$ Sinabi ni Heidegger, "The unconcealing of the concealed into unconcealment is the very presencing of what is present. We call this the Being of beings." Martin Heidegger, "Logos," in Early Greek Thinking, trans. by David Farrell Krell and Frank Capuzzi (San Francisco: Harper San Francisco, 1984), 64.

(C) 2013 Emmanuel C. de Leon

http://www.kritike.org/journal/issue 13/de leon december2013.pdf

ISSN 1908-7330

(cc) BY-NC 
kasaysayan ng Kanluraning Metapisika ay eksklusibong nagwawaldas ng oras sa paglalantad lamang ng Sein at mistulang kapalaran nitong malimutan ang essensya ng katotohanan ng Sein bilang aletheia. Ang tanong ngayon, saan nga ba nanggaling ang mapanganib na pagkalimot sa katanungan sa Sein?

Ang nakahain na tanong ay mayroong tatlong implikasyon. Una, kailangan natin galugarin nang masinsinan kung paano mismo nagtanong si Heidegger sa tunay na pinagmulan ng pagkalimot sa Sein. Pangalawa, mistulang may pagdududa si Heidegger kung may kakayahan ba talaga ang nagtatanong na Da-sein na matakasan ang pagiging makakalimutin. At pangatlo, na may relasyon sa pangalawa, pinupunto nito ang isang pagtatama ng katungkulan ng Da-sein bilang pala-isip sa kasalukuyang teknolohikal na sitwasyon. ${ }^{6}$

Kung taimtim nating pakikinggan ang pagmumuni muni ni Heidegger mula sa pagkaunawa ng mga sinaunang Griyego sa orihinal na kahulugan ng Sein, mapapansin natin ang ugnayan ng Sein (bilang phusis) at katotohanan (bilang aletheia). ${ }^{7}$ Subalit, ang kasaysayan ng kanluraning metapisika ay naka-sentro sa pagtatanong ng pagka-ano (mukha ng paglalantad) ng Seindes at mistulang kapalaran ${ }^{8}$ nitong makalimutan ang presensya ng katotohanan ng Sein bilang pangyayaring lantaran-pagtatago (aletheia). Hindi ito isang konsepto, sa pagkamalay ni Heidegger, na

${ }^{6}$ Sa section $44 \mathrm{ng}$ Being and Time, inusisa ni Heidegger ang tunay na relasyon ng katotohanan at Sein mismo. Sa pamamagitan ng paghimay sa tradisyunal na pag-unawa sa katotohanan, naliwanagan si Heidegger sa makapangyarihang ginagampanan ng Da-sein sa pagtatanong sa Sein. Gayunpaman, nilalagom ni Heidegger na, "[t]he Being of truth is connected primarily with Dasein. And only because Dasein is constituted by disclosedness (that is, by understanding), can anything like Being be understood; only so is it possible to understand Being." (BT, 272). Sa madaling salita, ang Heidegger ng Being and Time ay kumbinsido sa kakayahan ng Da-sein na maunawaan ang kahulugan ng Sein (analytic of Dasein). Idinagdag pa niya, "...truth is only in so far as and as long as Dasein is" $(B T, 272)$. Pero, sa mga pahuling sinulat niya, may pagkiling na si Heidegger sa pagpapaubaya sa mismong Sein. Kailangan magbigay ng abiso sa kaibahan ng Later Heidegger upang maging mas malinaw at magalang ang pagsunod natin sa kaisipan ni Heidegger.

7 Pinapaburan ni Heidegger ang kahulugan ng katotohanan bilang aletheia, ang "lantaran-pagtatago," kaysa sa modernong kahulugan nito na nakabatay sa correspondence. Sa modernong panahon, masasabing totoo ang isang sinabi, halimbawa "Pula ang kulay ng kotse ni Marcial," kung ang pinatutungkulan nito ay tunay na kotseng kulay pula na pag-aari ng isang taong nagngangalang Marcial. Walang masama sa ganitong klase ng pagtingin sa katotohanan. Subalit, para kay Heidegger, hindi ito ontolohikal at samakatuwid hindi mahalaga sa kanyang proyekto ng pagtatalas ng katanungan sa Sein.

8 "Or does it belong to the essential destiny of metaphysics that its own ground withdraws from it because in the rise of unconcealedness its essential core, namely, concealedness, remains absent in favour of that which is unconcealed, which can thereby first appear as beings?" Martin Heidegger, "Introduction to 'What is Metaphysics?'” trans. by Walter Kaufmann, in Pathmarks, ed. William McNeill (Cambridge: Cambridge University Press, 1998), 280-281. 
nalalagyan ng konkretong depinisyon. Ang katotohanan ng Sein, ayon kay Heidegger, ay isang pangyayari. Ang Sein, sa orihinal na pagkaunawa dito, ay nag-nga-ngayon (present) sa kasaysayan. Upang maunawaan natin ito, pagnilayan muna natin kung ano ang ibig sabihin ni Heidegger sa "kasaysayan."

Sa ordinaryong pag-unawa sa kasaysayan, ito ay isang sistematikong pag-aaral ng mga tao, lugar, at mga pagkakataon na nangyari na sa nakaraan. Ang kasaysayan nga ay matatagpuan sa makakapal at maalikabok na mga libro na naglalaman ng makinis na kwento tungkol sa mga nangyari na upang magamit sa kasalukuyan at sa hinaharap. Sa madaling salita, kung nais mong malaman ang Kasaysayan ng Sein (sa ganitong ordinaryong pag-unawa sa kasaysayan), kailangan mo lamang bumisita sa library at maghalungkat ng mga libro tungkol dito. Ayon kay Heidegger, isa itong historiography (Historie) at hindi history (Geschicte) sa ontolohikal na pag-unawa. Ano ang kaibahan ng dalawa?

Sa depinisyon ni Heidegger, ang Historie ay isang syentia ng pagtitipon ng mga factum (mga bagay na nasa harapan mo; nakahandang siyasatin). Para maipaliwanag ang kaibahan nito sa Geschicte, tinatanong ni Heidegger, "Ano nga ba ang ibig sabihin ng kasaysayan bilang disiplina?"9 Upang maipaliwanag ito, pinatawan niya ng depinisyon ang Historie bilang tiyakang pagpapaliwanag ng nakaraan sa perspektibo ng saysay nito sa kasalukuyan at hinaharap. Samakatuwid, ang mga bagay bagay ay ipinapalagay na pwedeng kwentahin at isaayos upang magamit at mamanipula. ${ }^{10}$ Ang "tiyakang pagpapaliwanag" na ito ng nakaraan ay nanggagaling sa kahiligang ikulong sa mababagsik na depinisyon ang mga bagay bagay. Tinitingnan nito ang nakaraan bilang mga artefact na maaaring kwentahin para magamit sa hinaharap. Samakatuwid, ang kasaysayan, sa ganitong pagtingin, ay ang mga nangyari na.

Ang kasaysayan bilang Geschicte, sa kabilang banda, ay may kaugnayan sa kapalaran o tadhana. Galing ito sa salitang Aleman na Schicken na nangangahulugang "ipadala." Sa ganitong pagtingin, ang kasaysayan ay mayroong buhay-na-daloy at ang nakaraan ay nasa kasalukuyan pa rin sa kanyang paglalantad. Para sa mga maka-Geschicte na kasaysayanador, ang mga pangyayari sa nakaraan at patuloy pa ring nangyayari sa kasalukuyan. Ang kasaysayan, sa ontolohikal na pagkaunawa ni Heidegger, ay nangyayari. Isa itong pangyayari ng

\footnotetext{
9 Tingnan sa Martin Heidegger, Contributions to Philosophy (From Enowning), trans. by Parvis Emad and Kenneth Maly (Indianapolis: Indiana University Press, 1999), 347. (Sa mga susunod, ang mga halaw sa kathang ito ay ilalagay ng $C P$ ).

${ }^{10}$ Sa kanyang Enowning, tahasan niyang ipinaliwanag ang Historie, “The ascertaining explaining of the past from within the horizon of the calculative dealings of the present. Beings are hereby presupposed as what is orderable, producible, and ascertainable" $(C P, 347)$.
}

(C) 2013 Emmanuel C. de Leon http://www.kritike.org/journal/issue 13/de leon december2013.pdf ISSN 1908-7330 
paglalantad at pagtatago ng Sein. ${ }^{11}$ Samakatuwid, kung tayo ay magtatanong ng mismong kasaysayan ng Sein, ang kasaysayan bilang disiplina ay hindi nababagay. Maaaring may gamit ang ontic na pamamaraan ng Historie, pero kung tayo ay nagtatanong tungkol sa talagang kasaysayan ng Sein, isang magalang na pagkikinig sa mismong pinakikinggan (sa mismong Sein) ang dapat nating pagtuunan ng pansin. Ito ang "nakalimutan" ng tao sa pag-usbong ng mapag-kwentang pag-iisip. Inaanyayahan tayo ni Heidegger na tingnan ang kasaysayan ng Sein sa mismong essensya nito.

Dahil sa pagkaunawa ni Heidegger sa katotohan ng Sein bilang pangyayari na "lantaran-pagtatago" sa iba't ibang yugto ng kasaysayan, nagmuni muni siya sa uri ng paglalantad ng Sein sa modernong panahon, ang panahon ang makabagong teknolohiya.

\section{Ang mga Katanungan Tungkol sa Teknolohiya}

Sa kanyang sanaysay na "Die Frage nach Der Technik" ("Mga Katanungan Tungkol sa Teknolohiya") matiyagang nagmuni muni si Heidegger sa esensya ng teknolohiya. Ginalugad niya ang dimensyon ng teknolohiya hindi upang kalkalin at manipulahin ito nang sa ganun ay makabuo ng isang konkretong patakaran tungkol sa takbo ng mga makina. Sa halip, ayon kay Heidegger, ang gawaing ito ay isang bukas na pakikipagtagpo sa Sein.12 Ano ang pag-unawa ni Heidegger sa teknolohiya? At, bakit mahalaga ang mga katanungan ni Heidegger tungkol sa teknolohiya?

Kaugnay ng pagkagising ni Heidegger sa katotohanan ng Sein bilang "lantaran-pagtatago" sa kasaysayan (para maging mas sakto: Ang Sein ay Kasaysayan), natuon ang kanyang pag-iisip (Denken) sa esensya ng teknolohiya. Sa karaniwang pananaw, ang teknolohiya ay isang gamit o instrumento ng tao upang maisakatuparan ang kanyang gustong mangyari. Masasabi nga raw na ang teknolohiya ay naka-base sa patakaran ng sanhi at bunga (cause and effect). Dito mismo sa ordinaryong konsepto ng teknolohiya bilang kasangkapan o instrumento nagdududa si Heidegger. Kaya nga, inusisa muna niya ang orihinal na kahulugan ng "kadahilanan"

11 "Being as en-owning is history. It is from this perspective that what is ownmost to history must be determined, independently of the representation of becoming and development, independently of the historical [as discipline] observation and explanation" (CP, 348).

${ }^{12}$ Kaugnay dito, sinabi ni Heidegger, "The Relationship will be free if it opens our human existence to the essence of technology." Martin Heidegger, The Questions Concerning Technology and Other Essays, trans. William Lovitt (San Francisco: Harper \& Row, 1977), 3. (Sa mga susunod, ang mga halaw sa kathang ito ay ilalagay ng QCT).

(C) 2013 Emmanuel C. de Leon http://www.kritike.org/journal/issue 13/de leon december2013.pdf ISSN 1908-7330 
(causality). ${ }^{13}$ Sa kanyang pag-uusisa, natagpuan niya sa mga sinaunang Griyego ang orihinal na paggamit sa "kadahilanan" bilang aiton o "paglikha" na may kaugnayan sa kahulugan ng poeisis (pagkatha-ng-isangbagay). Pero napansin ni Heidegger na wala sa apat na uri ng "dahilan" (material, formal, efficient, at final cause) ang kakayahang "lumikha" ng isang bagay. Maging ang causa efficeins, ayon kay Heidegger, ay hindi maituturing na siyang lumikha sa isang bagay. Kung mataimtim ang pagsama natin sa pagmumuni muni ni Heidegger, maririnig natin na sinasabi niyang mayroon pang isa na nagdudulot at pinagkakautangan ng pagka-Sein labas sa apat na "dahilan."14 Pero, ano ang kaugnayan nito sa mga katanungan tungkol sa teknolohiya? Kung ang pamimilosopiya ni Heidegger ay nakatuon sa pagpapalinaw ng Seinsfrage, bakit siya nagtatanong tungkol sa teknolohiya?

Ang teknolohiya ay hindi lang basta instrumento upang makuha ang ninanais ng tao. Sa mas orihinal na kahulugan ng teknolohiya bilang techne, isa itong uri ng paglalantad na napapaloob sa kahulugan ng poeisis. ${ }^{15}$ Samakatuwid, nailantad ni Heidegger ang kahulugan ng teknolohiya na malayong malayo sa maka-makinang kahulugan nito. Paano nga ba nagyayari (naglalantad) ang teknolohiya sa makabagong panahon?

Sa pagmumuni muni ni Heidegger sa esensya ng teknolohiya, naliliwanagan siya sa kaakibat na panganib ng paglalantad ng makabagong teknolohiya na malayong malayo sa poeisis. Ang modernong teknolohiya ay nauunawaan sa makabagong panahon bilang "mapagpilit" [Herausfordern]. Pinipilit nito ang mundo na maglabas ng bagay bagay para magamit ng tao sa kasalukuyan o sa hinaharap. Ang tao, bilang suheto na tinitingnan ang mundo bilang bestand (o bagay na nasa harapan para gamitin o ireserba), ang siyang tumatayong mapilit at manggagamit.

Sa kabila ng ginagampanan ng tao sa modernong panahon bilang mapilit na manggagamit, hindi ibinabato ni Heidegger ang sisi sa mga tao. Ang mga "katanungan" ni Heidegger tungkol sa teknolohiya ang naglantad ng hindi matatakasang gapos na tinatawag niyang Enframing. Sa loob ng kondisyon ng Enframing, ayon kay Heidegger, ang tao ay walang magagawa kundi tingnan ang mga bagay sa harapan niya bilang gamit

${ }^{13}$ Kaugnay sa pagmumuni muni ni Heidegger sa "kadahilanan," sinabi niya, “"For a long time we have been accustomed to representing cause as that which brings something about" (QCT, 7).

14 "Causa, casus, belongs to the verb cadere, "to fall," and means that which brings it about that something falls out as a result in such and such a way.... What we call cause [Ursache] and the Romans call causa is called aiton by the Greeks, that to which something else is indebted [das, was ein anderes verschuldet]" (QCT, 7).

15 "Technology is therefore no mere means. Technology is a way of revealing. If we give heed to this, then another whole realm for the essence of technology will open itself up to us. It is the realm of revealing, i.e., of truth" $(Q C T, 10)$.

(C) 2013 Emmanuel C. de Leon

http://www.kritike.org/journal/issue 13/de leon december2013.pdf

ISSN 1908-7330

(cc) BY-NC 
kasama na rito ang kanya mismong sarili. Nakita ni Heidegger sa puntong ito ang tunay na panganib. ${ }^{16}$ Sa unang tingin, ang tao ang siyang nagmamaniobra at sinusunod ng mga bagay. Ngunit sa mas malalimang pagtingin, ang tao mismo ay nasa bingit ng panganib na maibaba sa abang kalagayan ng mga bagay na nakahandang gamitin sa anumang oras. Paano tayo makakatakas sa panganib na dulot ng essensya ng modernong teknolohiya?

Sa pag-unawa ni Heidegger, hinding hindi natin matatakasan ang panganib na ito at nasa mismong panganib ang posibilidad ng pagkaligtas. Sa halip, isang kalagayan ang dapat yakapin ng tao kung gusto niyang makawala sa gapos at panganib ng Enframing. Hindi ito tahasang pinangalanan ni Heidegger. Tinawag lamang niya ito sa isang bansag na Ereignis kung saan isa itong kalagayan ng hindi palagay o laging handa sa posibilidad at panganib na tingnan ang mga bagay bagay bilang simpleng gamit lamang. Sa ganitong uring pananaw, walang magagawa ang pag-iisip na syentipiko. Maging ang tradisyunal na pilosopiya, sa pagtingin ni Heidegger, ay natatapos na kung gusto nating manatili sa presensya ng Sein. Sa Ereignis, ang gagampanan lamang ng tao ay ang maghintay sa pagtalab ng Sein. Ito nga ang sinasabing pagdiriwang sa Sein sa katapusan ng pilosopiya.

\section{Ang Kapalaran ng Pag-iisip ni Heidegger: Isang Aporia}

Sa takbo ng pag-iisip ni Heidegger, ang kaliwanagan sa katotohanan ng Sein bilang naglalandtad-nagtatago sa mismong kasaysayan ay tumuturo sa pagtatanong kung ano nga ba ang papel ng tao (Dasein) sa pagtatanong sa Sein. Dahil sa masinsinang pagmumuni muni, para kay Heidegger, ang tao nga ay napapaloob sa kasaysayan ng Sein. ${ }^{17}$ Taliwas ito sa tema ng makabagong panahon na naniniwala sa tao bilang sentro ng santinakpan. Kung nais ng taong mamuhay na malapit sa Sein, kailangan niyang matutunang maghintay ng may pananabik sa "pagtatakda" ng Sein. Samakatuwid, ang wakas ng pilosopiya, bilang uring pag-iisip na nagdidiriwang sa mga bungang-isip, ay pinamamalita na ni Heidegger. Sa puntong ito, ang kapalaran ng pag-iisip ni Heidegger ay ang pagkatagpo sa

16 Sa puntong ito, nakikita na natin na para kay Heidegger ang pagtingin natin sa mga bagay na nakapaligid sa atin ay siya ring pagtingin natin sa ating sarili.

17 "Who accomplishes the challenging setting-upon through which what we call the real is revealed as standing-reserve? Obviously, man. To what extent is man capable of such a revealing? Man can indeed conceive, fashion, and carry through this or that in one way or another. But man does not have control over unconcealment itself, in which at any given time the real shows itself or withdraws. The fact that the real has been showing itself in the light of Ideas ever since the time of Plato, Plato did not bring about. The thinker only responded to what addressed itself to him" (QCT, 18). 
isang aporia. Sa harap ng isang dead-end, hindi na nga makaka-abante ang isang palaisip. Wala na siyang magagawa kundi ang isuko (Gelassenheit) ang kanyang sarili, ang "mag-isip" labas o lampas sa pilosopiya. Sa katapustapusan, inilalantad ni Heidegger ang orihinal na katayuan ng tao, pero hindi siya nagbibigay ng reseta kung paano nga mamuhay bilang tao. Hinahayaan niyang bukas ang larangan ng buhay para sa malayang pakikipagrelasyon ng tao sa lahat lahat. Hindi niya sinasagot ang mailap na tanong sa Sein hindi lang dahil walang kongkretong sagot dito, kundi dahil isa itong misteryo na hinding hindi mahuhuli sa isang konsepto. Gayunpaman, sa ating pagsunod sa pag-iisip ni Heidegger, ginigising tayo sa ating mga kinahuhumalingan sa makabagong panahon.

Sa kabilang banda, maaari rin namang sabihin na ang pilosopiya ni Heidegger ay isang panawagan na humanap ng mga panibagong daan palabas sa tradisyunal na patakaran ng Kanluraning Pilosopiya. Gumamit tayo ng paghahalimbawa. Ang pilosopiya ni Heidegger ay maihahalintulad sa karanasan ng pagkaligaw sa gitna ng isang masukal na kagubatan. Kung ikaw ay naliligaw, hinahamon ka ng iyong sitwasyon na hanapin ang tamang daan o hindi kaya ay gumawa ng panibagong daan palabas ng kagubatan. Kaya nga, masasabi natin na ang mga natagpuan ni Heidegger sa kanyang pagmumuni muni sa mismong naglalantad-nagtatagong Sein ay nag-iiwan sa atin ng panibagong gawain-ang ipagpatuloy ito sa paraang bago o mas mainam sigurong sabihing sa paraang orihinal at nababatay sa ating mismong katayuan bilang tao.

\section{Isang Paglalagom}

Sa puntong ito, mistulang ipinamamalita na ni Heidegger ang katapusan ng pilosopiya. Ang Kanluraning Metapisika na nagwawaldas ng oras sa katotohanan ng Sein ay dumating na sa dulo at wala nang mapupuntahan pa. Kung totoo ang sinasabi ni Heidegger, ano na ang kapalaran ng tao sa katapusan ng pilosopiya? Anong klaseng kalagayan ang dapat paghandaan ng tao sa katapus-tapusan ng pilosopiya? Sa isang mapagnilay na paraan, sinagot ni Heidegger ang katanungang ito nang buong ingat,

Let me respond briefly and somewhat ponderously, but from long reflection: philosophy will not be able to effect an immediate transformation of the present condition of the world. This is not only true of philosophy, but of all merely human thought and endeavour. Only a god can save us now. The sole possibility that is left for us is to prepare a sort of

(C) 2013 Emmanuel C. de Leon

http://www.kritike.org/journal/issue 13/de leon december2013.pdf

ISSN 1908-7330

(cc) BY-NC 
readiness, through thinking and poetizing, for the appearance of the god or for the absence of the god in the time of foundering (Untergang); for in the face of the god who is absent, we founder. ${ }^{18}$

Ang paghihintay sa pagdating ng "diyos" sa lugmok na kalagayan (foundering stage) ng modernong panahon ay nangangahulugan ng paglalagay sa sarili sa isang hindi palagay na sitwasyon. Isang poetic dwelling, ang terminolohiyang ginamit ni Heidegger, ang kailangan pagaralan ng tao bilang kanyang saloobin sa kasalukuyang panahon. Ang isang makata (makatha), isang mulat sa kung anumang nagpapakita, ay patuloy na naaakit at lumalapas sa nakikita. Tinatawag ito ni Heidegger na "other thinking." Isang pag-iisip o mentalidad na malayong malayo sa makamakina at mapag-kwentang pag-iisip. Hindi ito nangangahulugang titigil na ang tao at matutulog na lamang sa pansitan habang naghihintay. Isang malikhaing pagkatha na umuusbong sa mapagpaubayang pagninilay ang kailangang yakapin ng tao.

Sapagkat hindi tayo inaakay ng mapag-kwentang pag-iisip sa isang ontolohikal na katotoohan, ipinapakita ni Heidegger sa kanyang mambabasa ang kahalagahan ng "pagpapaubaya" at mapagnilay na pagiisip. Ang ganitong kalagayan ang magdadala sa tao pabalik sa kanyang sarili mismo. Samakatuwid, isang may pananabik na pagbalik sa sarili ang itinuturo ni Heidegger. ${ }^{19}$ Dahil sa tinatawag ni Heidegger na "Fallenness" sa Being and Time, nakalimutan nga ng tao ang tunay niyang katayuan bilang tao. Ito ang sinasabi ni Heidegger na malikhaing pagbalik (creative retrieval) sa orihinal essensya ng tao.

May kahirapan ang paggawa ng isang paglalagom sa pilosopiya ni Heidegger dahil maging siya mismo ay walang kahiligan sa paglalahat ng mga bagay bagay. Para sa kanya, ang katotohanan ay hindi natutulog sa pansitan, mailap, at hinding hindi maikukulong sa iisang kahulugan. Ang istilo ng pamimilosopiya ni Heidegger ay nakatuon sa pagdanas ng mga bagay upang sa ganun ang mismong pag-iisip ang mag-akay sa kanya sa isang kahulugan. Samakatuwid, ang uring pag-iisip ni Heidegger ay nakasentro sa pagmumulat sa parte ng mga mambabasa na makinig at mag-isip sa mas orihinal na paraan, sa isang uri ng pag-iisip na hindi tayo ang bida. Ang muling pagkakatuklas ni Heidegger sa kakanyahan (inherent nature) ng Sein, na "lantarang pagtatago" ng sarili sa kasaysayan, ay nag-aanyaya sa

\footnotetext{
${ }^{18}$ Martin Heidegger, "Only a God Can Save Us: Der Spiegel's Interview with Martin Heidegger," in The Heidegger Controversy, ed. Richard Wolin (Cambridge: MIT Press, 1993), 107.

${ }^{19}$ Sanabi ni Heidegger, "Travelling in the direction that is the way toward that which is worthy of questioning is not an adventure but a homecoming." "Letter on Humanism," 271272.
} 


\section{PAGTATALAS SA TANONG SA SEIN}

atin na danasin ito ayon sa mismong kakanyahan ng Sein. Dahil dito, inaanyayahan tayo ni Heidegger na tumanaw pabalik sa mismong sarili natin. Maaari nga nating sabihin, kung talagang tama si Heidegger, na ang pagkalimot sa Sein ay siya ring pagkalimot ng mga tao sa kanyang katayuan bilang mortal. Sa madaling salita, ang krisis ng makabagong panahon ay produkto nga ng pagkalimot sa tanong sa Sein.

Department of Philosophy, University of Santo Tomas, Philippines

\section{References}

Heidegger, Martin, "Introduction to 'What is Metaphysics?"” trans. by Walter Kaufmann, in Pathmarks, ed. William McNeill (Cambridge: Cambridge University Press, 1998).

"Letter on Humanism," in Basic Writings, ed. David Farrell Krell (USA: HarperCollins Publishers, 1993).

"Logos," in Early Greek Thinking, trans. by David Farrell Krell and Frank Capuzzi (San Francisco: Harper San Francisco, 1984).

"On the Essence of Truth," trans. by John Sallis, in Pathmarks, ed. William McNeill (Cambridge: Cambridge University Press, 1998).

"Only a God Can Save Us: Der Spiegel's Interview with Martin Heidegger," in The Heidegger Controversy, ed. Richard Wolin (Cambridge: MIT Press, 1993).

Being and Time, trans. by Joan Stambaugh (New York: State University of New York Press, 1996).

Contributions to Philosophy (From Enowning), trans. by Parvis Emad and Kenneth Maly (Indianapolis: Indiana University Press, 1999). 